\title{
Phase and Amplitude Calibration of Rotating Equispaced Circular Array for Geostationary Microwave Interferometric Radiometers- Theory and Methods
}

\author{
Xi Guo, Student Member, IEEE, Adriano Camps, Fellow, IEEE, Hyuk Park, Senior Member, IEEE \\ Hao Liu, Cheng Zhang, Member, IEEE, Ji Wu, Fellow, IEEE
}

\begin{abstract}
The calibration strategy for synthetic aperture interferometric radiometers based on noise injection has been successfully demonstrated in the Microwave Imaging Radiometer with Aperture Synthesis (MIRAS) instrument onboard the Soil Moisture and Ocean Salinity (SMOS) mission of the European Space Agency (ESA). With the emerging demands of increasing both array size and frequency bands, the internal calibration hardware becomes more difficult to implement. Although several external calibration methods have been proposed, the so-called phase ambiguity problem was not carefully considered. In this paper, a novel calibration strategy for both phase and amplitude calibration based on redundant space calibration is proposed, which utilizes the unique structure of equispaced circular array and rotational sampling strategy. The original irregular phase ambiguity degenerates to the fixed $\pi$-ambiguity, where only two possible solutions to phases are introduced. Both phase and amplitude of visibility can be simultaneously calibrated by the proposed strategy, and no cooperative calibration target is needed.
\end{abstract}

Index Terms-Microwave radiometry, amplitude calibration, phase calibration, redundant baselines, circular array, rotational sampling, synthetic aperture interferometric radiometer.

\section{INTRODUCTION}

$\mathbf{P}$ ASSIVE microwave radiometry has provided unprecedented observations of the Earth, and its importance has been demonstrated to understand the Earth's environment and develop numerical weather prediction. With the design of observational frequency bands and radiative transfer models,

X. Guo is with the University of Chinese Academy of Sciences, Beijing 100049, China, and also with the Key Laboratory of Microwave Remote Sensing, National Space Science Center, Chinese Academy of Sciences, Beijing 100190, China (email: xi_guo@hotmail.com).

A. Camps and H. Park are with the Department of Signal Theory and Communications, Universitat Politècnica de Catalunya, Unidad de Excellencia María de Maeztu - CommSensLab, and Universitat Politècnica de Catalunya - BarcelonaTech, and IEEC/CTE-UPC, 08034 Barcelona, Spain (emails: camps@tsc.upc.edu; park.hyuk@upc.edu).

H. Liu, C. Zhang, J. Wu are with the Key Laboratory of Microwave Remote Sensing, National Space Science Center, Chinese Academy of Sciences, Beijing 100190, China (emails: liuhao@mirslab.cn; zhangcheng@mirslab.cn; wuji@nssc.ac.cn).

NSSC's contribution was supported by the National Natural Science Foundation of China under Grant 41675035, the special Fund for Chinese Meteorological Research in the Public Interest under Grant GYHY201506023, and Beijing Science Project under Grant Z161100002616033. UPC's contribution was supported by the Spanish Ministry of Science, Innovation and Universities, "SPOT: Sensing with Pioneering Opportunistic Techniques", Grant RTI2018-099008-B-C21/AEI/10.13039/501100011033. the corresponding physical parameters can be retrieved by passive microwave observations [1], for example, soil moisture and ocean salinity $(1.4 \mathrm{GHz})[2],[3]$, atmospheric temperature $(53 \mathrm{GHz})$ [4], humidity profiles $(183 \mathrm{GHz})$ [5], etc.

Since high spatial resolution observation is required for more precise applications, interferometric radiometry has been considered as a competitive technique to achieve the requirements. Compared to the conventional real aperture technique, synthetic aperture interferometric radiometers employ multiple small antennas to create an equivalent virtual aperture by aperture synthesis [6]-[8]. The application of interferometric radiometry on Earth observation has been successfully demonstrated by Microwave Imaging Radiometer by Aperture Synthesis (MIRAS), the single payload of the Soil Moisture and Ocean Salinity (SMOS) mission [9]. Since then, several future synthetic aperture interferometric payloads have been under development, especially for geostationary microwave sounders, for instance, GeoSTAR proposed by the Jet Propulsion Laboratory, NASA [10], [11]; GAS proposed by the European Space Research and Technology Centre, ESA [12]; and GIMS proposed by the National Space Science Center, Chinese Academy of Sciences [13], [14].

As Low-Earth-Orbit (LEO) microwave radiometers have been operated since the 1970s, the weather forecast accuracy has been significantly improved. However, LEO satellites suffer from limited field of view ( $2000 \mathrm{~km}$ swath) and low temporal resolution ( $\sim 12$ hours). Rapidly evolving weather phenomena typically have a life cycle of 30-60 minutes, such as strong convective weathers. Not only continuous monitoring is required for large-scale tropical cyclones, but also a large spatial coverage. The combination of geostationary orbit (GEO) and synthetic aperture interferometry makes it promising to meet both requirements. Unlike the real aperture radiometer, synthetic aperture interferometric radiometers directly measure the spatial frequency components of the brightness temperature distribution by numerous baselines simultaneously, which allows both the desired spatial and temporal resolution. The instruments under development for future GEO passive microwave missions will provide a full coverage of the Earth disk with spatial resolutions of $50 \mathrm{~km}$ at $53 \mathrm{GHz}$ [14] and $25 \mathrm{~km}$ at $183 \mathrm{GHz}$ [15], every 15 minutes. Moreover, they can be further improved by implementing deployable antenna structures [16] and super-resolution algorithms [17], [18]. 
Calibration is the key to synthetic aperture interferometric radiometry. Sophisticated calibration strategy has been designed and implemented successfully on MIRAS by the means of noise injection method [19], [20]. Centralized noise injection is impractical for on-orbit mission, since its hardware complexity grows quadratically with the number of receiving elements. Therefore, a distributed approach is conceived [21]. For synthetic aperture interferometric radiometers, if high spatial resolution is required by the scientific objectives, the number of receiving elements will be inevitably large. Although electronic technology has been advanced considerably after the development of SMOS, the internal calibration system is still challenging to be implemented on the large-scale array, especially at the high frequency bands $(53 \mathrm{GHz}$ and $183 \mathrm{GHz}$ ).

The external calibration is widely implemented in observational astronomy, which is referred to as "self-calibration" and "redundant baseline calibration" [22]. Self-calibration was firstly introduced in radio astronomy [23], it basically minimizes the deviation of the observed visibility from a model of the source visibility, where complex gain factors are the actual unknowns to be solved. The model of the observed scene should be astronomically reasonable, which means possible constrains and specific structures are required in visibility modeling. However, self-calibration fails when either the signal-to-noise ratio is too low or the source is too complex [24]. On the other hand, the basic idea of redundant space calibration is using the internal consistency of redundant visibility measurements; thus, complex gain factors and true visibilities can be solved without any other dedicated calibration hardware or target-based model [25]. Recently, numerous redundant baseline calibration methods have been developed and implemented on the instruments for $21 \mathrm{~cm}$ cosmology, where the sky-based calibration is especially difficult to be applied [26]. And several interferometric instruments for 21 $\mathrm{cm}$ cosmological observations have been designed and manufactured to have redundant measurements, such as MITEoR [27], PAPER [28], and HERA [29]. In optical astronomy, several phase-retrieval methods similar to redundant baseline calibration have been successfully developed for bispectrum imaging [30], [31]. In both research fields of astronomy, the problem of the so-called phase ambiguity is found to be unavoidable, and several techniques have been proposed to solve it [32]-[35].

The external calibration for synthetic aperture interferometric radiometers has been considered as an auxiliary or an alternative to the classical internal calibration. Redundant space calibration (RSC, as same as the redundant baseline calibration in radio astronomy) has been proposed for MIRAS [36], but it is limited by the residual error amplification along the arms [37], [38]. A hybrid calibration method was developed to downscale the noise injection network, which combines RSC and reduced noise injection [39]. Similar to model-based calibration, phase calibration by the ancillary observation targets has also been proposed. The point-sourcelike targets are considered as external calibration sources due to their straightforward visibility models, such as the Sun [40] and point-source beacons [41], [42]. As the rotational sampling strategy is developed for the sparse array to compensate sparse sampling in the $u-v$ domain [13], [43], several phase calibration techniques have been designed to use the inherent nature of the rotationally sampled visibilities [44], [45]. Even though phase calibration has drawn great attention in the previous researches, there is not any closed-form solution for the problem of phase ambiguity, which is critically emphasized by radio astronomers. Insufficient consideration of phase ambiguity results in a limited application of the phase calibration, and further phase-unwrapping method needs to be developed.

In this paper, a novel calibration strategy for equispaced circular array based on redundant space calibration and rotational sampling is presented, it aims to calibrate both phase and amplitude simultaneously by utilizing its strong internal self-consistency, and the ambiguity of the retrieved phases is discussed in detail.

This paper is organized as follows. In Section II, the basics of visibility function and calibration model are reviewed, and the equispaced circular array is introduced. Section III describes the systems of calibration equations established by the rotational sampling strategy, and different ways to build them. Finally, Section IV and Section V present the techniques of phase retrieval and amplitude retrieval by the corresponding system of calibration equations, respectively.

\section{PRELIMINARIES}

\section{A. Visibility Function and Calibration Model}

In synthetic aperture interferometry, the spatial frequency components of the brightness temperature image are measured by the complex cross-correlation of the signals collected by every pair of elements in the array, which is the so-called visibility function. Without considering system imperfections, the samples of visibility function are given by [46]

$$
V(u, v)=\iint_{\xi^{2}+\eta^{2} \leq 1} T^{\prime}(\xi, \eta) \cdot \tilde{r}\left(\frac{u \xi+v \eta}{f}\right) \cdot e^{-j 2 \pi(u \xi+v \eta)} d \xi d \eta
$$

where $(u, v)=\left(x_{k}-x_{l}, y_{k}-y_{l}\right) / \lambda$ are the spatial frequency determined by the antenna separation normalized to the wavelength $\lambda,(\xi, \eta)=(\sin \theta \cos \phi, \sin \theta \sin \phi)$ are the direction cosines with respect to the $x$ and $y$ axes, $\tilde{r}$ is the fringe-washing function that accounts for spatial decorrelation effects, and $T^{\prime}(\xi, \eta)$ is the so-called modified brightness temperature

$$
T^{\prime}(\xi, \eta)=\frac{T_{B}(\xi, \eta)-T_{r}}{\sqrt{1-\xi^{2}-\eta^{2}}} \cdot \frac{F_{n k}(\xi, \eta) \cdot F_{n l}^{*}(\xi, \eta)}{\sqrt{\Omega_{k} \cdot \Omega_{l}}}
$$

where $T_{B}(\xi, \eta)$ is the brightness temperature of the scene, $T_{r}$ is the physical temperature of receivers, $\sqrt{1-\xi^{2}-\eta^{2}}$ is the obliquity factor, $F_{n k, l}(\xi, \eta)$ are the normalized antenna voltage patterns of the elements in the array, $(\cdot)^{*}$ is the complex conjugate operation, and $\Omega_{k, l}$ are the antenna solid angles.

In the ideal case, the spatial decorrelation effects are negligible $(\tilde{r} \approx 1)$, all antenna patterns are equal $\left(F_{n k}=F_{n l}=F_{n}\right)$, the relationship between the visibility function and the modified brightness temperature reduces to the Fourier transform relationship. Thus, several image reconstruction methods have 
been developed based on the fast Fourier transform and the specific structure of $u-v$ sampling grid [47]-[49]. An extension of (1) to the full-polarimetric case is given in [50] and the effect of the antenna pattern frequency dependence is presented in [51].

Without loss of generality, we will focus on the single polarization case as given by (1). Before calibration, the uncalibrated visibility is denoted by the complex cross-correlation of two pairs of signals of the receiving channels, and the corresponding measured signal $s_{k}$ of $k^{\text {th }}$ element can be expressed by

$$
s_{k}(t)=\gamma_{k} \cdot x_{k}(t)+n_{k}^{s}(t)
$$

where $\gamma_{k}$ is the complex gain factor of the receiving channel, $x_{k}(t)$ is the ideally observed signal that would be measured with a perfect receiver, and $n_{k}^{s}(t)$ is the instrumental noise of the receiving channel.

Assuming that $x_{k}(t)$ and $n_{k}^{s}(t)$ are uncorrelated and the non-separable complex gain factor is negligible, the complex cross-correlation of the signals measured by a baseline (antenna pairs $k^{t h}$ and $l^{t h}$ ) can be expressed by

$$
\begin{aligned}
V_{k l}^{\mathrm{raw}}= & \frac{1}{2}\left\langle s_{k}(t) \cdot s_{l}^{*}(t)\right\rangle \\
= & \frac{1}{2} \gamma_{k} \gamma_{l}^{*}\left\langle x_{k}(t) x_{l}^{*}(t)\right\rangle+\frac{1}{2} \gamma_{k}\left\langle x_{k}(t) n_{l}^{s *}(t)\right\rangle \\
& +\frac{1}{2} \gamma_{l}^{*}\left\langle x_{l}^{*}(t) n_{k}^{s}(t)\right\rangle+\frac{1}{2}\left\langle n_{k}^{s}(t) n_{l}^{s *}(t)\right\rangle \\
= & \gamma_{k} \cdot \gamma_{l}^{*} \cdot V_{k l}^{\mathrm{id}}+n_{k l}^{c}
\end{aligned}
$$

where $\langle\cdot\rangle$ denotes time average within the integration time, $V_{k l}^{\text {id }}=\frac{1}{2}\left\langle x_{k}(t) \cdot x_{l}^{*}(t)\right\rangle$ is the ideal visibility that has the same definition in (1). As the signal and noise are uncorrelated, the second and third term on the right-hand side are equal to zero; and the last term is attributed to the additive zero-mean noise at the complex correlation level, which is denoted as $n_{k l}^{c}$.

The calibration of complex gain factors is conducted by phase calibration and amplitude calibration, respectively. For phase calibration, the corresponding noise-free calibration model can be written as

$$
\psi_{k l}^{\mathrm{raw}}=\phi_{k}-\phi_{l}+\psi_{k l}^{\mathrm{id}}
$$

where $\psi_{k l}^{\text {raw }}=\arg \left(V_{k l}^{\text {raw }}\right)$ is the phase of the measured complex correlation, $\psi_{k l}^{\text {id }}=\arg \left(V_{k l}^{\mathrm{id}}\right)$ is the phase of the ideal visibility, and $\phi_{k, l}=\arg \left(\gamma_{k, l}\right)$ is the phase of the complex gain factor, also denoted as the separable phase factor [36].

Similarly, the noise-free calibration model for amplitude calibration can be written as

$$
\left|V_{k l}^{\mathrm{raw}}\right|=g_{k} \cdot g_{l} \cdot\left|V_{k l}^{\mathrm{id}}\right|
$$

where $\left|V_{k l}^{\mathrm{raw}}\right|$ is the amplitude of the measured complex correlation, $\left|V_{k l}^{\text {id }}\right|$ is the amplitude of the ideal visibility, $g_{k, l}=\left|\gamma_{k, l}\right|$ is the amplitude of the complex gain factor, also denoted as the separable gain factor. Note that the products in (6) can be linearized by taking the natural logarithm

$$
\ln \left|V_{k l}^{\mathrm{raw}}\right|=\ln \left(g_{k}\right)+\ln \left(g_{l}\right)+\ln \left|V_{k l}^{\mathrm{id}}\right| .
$$

thus, the natural logarithm of the separable gain factors and the ideal visibility amplitudes become the direct unknowns to solve for.

\section{B. Basics of Equispaced Circular Array}

On an equispaced circular array, the spacings of adjacent antenna elements and the central angles formed by every pair of adjacent elements are identical for a given array configuration. There are three fundamental quantities to describe the geometry of an equispaced circular array: 1) the total number of elements $M ; 2$ ) the adjacent element spacing $d_{s}$; and 3) the radius of the circular array $R$. By the law of cosines, their relationship can be described as (see Fig. 1.)

$$
\cos \left(\frac{2 \pi}{M}\right)=\frac{2 R^{2}-d_{s}^{2}}{2 R^{2}}
$$

where $2 \pi / M=\theta_{c}$ is the central angle.

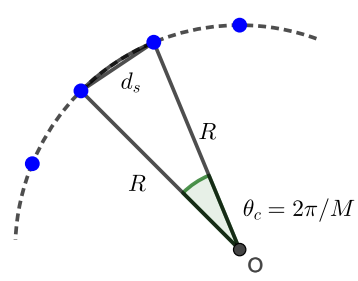

Fig. 1. Geometry of equispaced circular array.

Clearly, any two of these quantities can uniquely determine an equispaced circular array. From the perspective of synthetic aperture radiometer's design, these three quantities have practical meanings that describe the instrument from technical points of view. The radius of a circular array $R$ establishes the maximum baseline length, which indicates the instrument's spatial resolution. The adjacent element spacing $d_{s}$ determines the maximum radial sampling interval of visibilities in the $u$ $v$ domain, which can be used to indicate the alias-free field of view (AF-FOV) [13]. The total number of elements $M$ is an essential indicator of the system complexity, not only for hardware configuration but also for the implementation of calibration and image reconstruction algorithm. In addition, as the total number of elements determines the number of visibility samples, it further determines the radiometric sensitivity ${ }^{1}$ of the array designed [53].

\section{Visibility Samples}

Since the coordinates of visibility samples are defined by the relative distance of every two element pairs, the visibility sampling coverage formed by an equispaced circular array also has a circular shape. Fig. 2 shows the visibility samples and its Hermitian conjugate of a snap-shot measurement with an even/odd number of elements. The parity of the total number of elements leads to different redundancy factors of the visibility samples. For an even number of elements, most of the visibility samples have a redundancy factor of two, except for the visibility samples on the outermost circle. In contrast, for an odd number of elements, the central asymmetry results in no instantaneous redundancy.

\footnotetext{
${ }^{1}$ The GeoSTAR instrument has a radiometric sensitivity of $\sim 0.5 \mathrm{~K}$ with a 15-minute integration time [15]. And the radiometric performance of equispaced circular array has been analyzed, see [52] for details.
} 


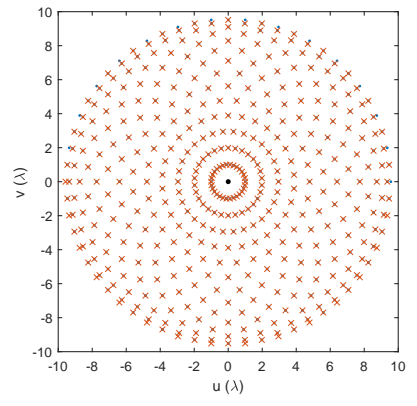

(a)

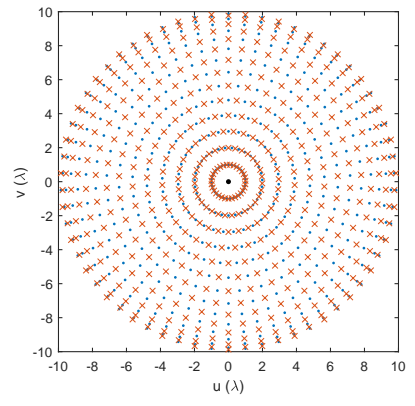

(b)
Fig. 2. Visibility samples of equispaced circular array for an instantaneous measurement. The overlap of original sample and its Hermitian conjugate denotes the redundancy of visibility sample at the current sampling position. (a) Even elements. (b) Odd elements.

In the circular direction, the visibility samples are equally distributed in a series of concentric circles, and the amounts of visibility samples on each concentric circle are identical. In the radial direction, the wavelength-normalized baseline lengths of visibilities on concentric circles (i.e., the radii of concentric circles in $u-v$ domain) can be described as a sine function

$$
L_{k}=2 R \cdot \sin \left(\frac{k \cdot \pi}{M}\right), \quad k=1,2, \ldots,\lfloor M / 2\rfloor
$$

where $R$ is the radius of the circular array, $M$ is the total number of elements, $\lfloor\cdot\rfloor$ denotes the floor function. In both cases, the visibility samples tend to be more densely packed in the central and edge part of sampling coverage [52].

Although the circular array formed by an odd number of elements has a two-fold higher sampling density in the circular direction than the array with an even number of elements, it also suffers from the side effects caused by the loss of instantaneous redundancy. The visibility sampling coverage will not be complete if any receiving channel's failure happens, which may further influence the performance of brightness temperature reconstruction.

As the equispaced circular array provides complete coverage of visibility function within the array size by snap-shot measurement, it does not require array rotation to cover the missing samples in $u$-v domain during the imaging period (for brightness temperature retrieval). In other words, array rotation is only required during the calibration period.

\section{System of CAlibration EQUations AND PROPERTIES OF ROTATIONAL SAMPLING}

The essence of redundant space calibration relies on using redundant measurements to build a system of calibration equations, then utilizing the self-consistency of redundant measurements to calibrate the instrument. One important way to evaluate redundant space calibration is to examine the relationship between the rank of the system of calibration equations and the number of unknowns. The degrees of freedom (i.e., the difference between the rank and the number of unknowns) determine whether redundant space calibration is feasible for the current array configuration because the calibration is carried out by solving the system of calibration equations, and the system parameters are denoted by the unknowns to be solved. Also, if absolute calibration is required, the degrees of freedom for the system of calibration equations have to be eliminated by other kinds of auxiliary calibration methods. Evaluations of the calibration equations and the properties of rotational sampling for the equispaced circular array are presented below.

\section{A. Snap-shot Measurement}

All possible antenna pairs (i.e., baselines) in array geometry view for a snap-shot measurement are illustrated in Fig. 3, for both even/odd number of elements $\left(M_{1}=6\right.$ and $M_{2}=7$, for instance). We define the baselines with identical length as the group of the baselines formed by the same relative separation of two elements, and they are also denoted by the same-length baseline group in the following analysis. It should be noted that the baselines in the same-length baseline group are not necessarily redundant in a snap-shot measurement, because the orientations of these baselines are not fully identical. As will be presented in Section III-B, they become completely redundant by the implementation of rotational sampling.

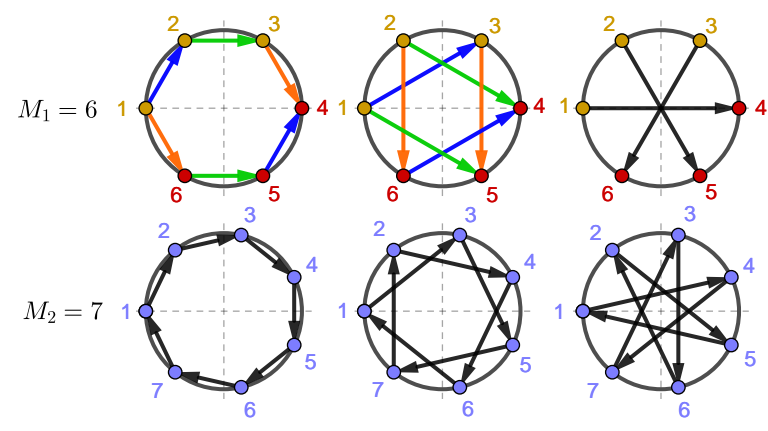

Fig. 3. Baselines formed by a snap-shot measurement. The groups of samelength baselines are shown in different columns, and the baselines in same color indicate the instantaneous redundancy in this group.

It is obvious that there is not any redundant measurement for the array with an odd number of elements, and the degrees of freedom in the system of calibration equations are far more than the number of unknowns, so that evaluation for this array configuration is omitted here.

For the array with an even number of elements, the instantaneous redundancy factor is 2 (shown in colors), except for the longest baselines (diagonal baselines). Since redundancy is needed as much as possible to reduce the number of unknowns, the longest baselines are not considered when building the system of calibration equations. Excluding the diagonal baselines, there are $M / 2-1$ groups of same-length baselines. In addition, each group contains $M$ baselines, so the number of equations is $M(M / 2-1)=M(M-2) / 2$. The number of ideal visibility terms is $M / 2$ for each group of same-length baselines, so the total number of ideal visibility terms is found to be $M / 2 \cdot(M / 2-1)=M(M-2) / 4$. Adding $M$ separable factors, the number of unknowns is found to be $M(M+2) / 4$. 
As it can be seen from (5) and (7), the formation of phase calibration equations and amplitude calibration equations is different $(+1$ and -1 coefficients appear in the phase calibration equation, while only the +1 coefficient exists in the amplitude calibration equation). Building the system of equations for both phase and amplitude calibration respectively, the corresponding relationship between the coefficient matrix rank and the number of unknowns can be summarized as:

1) Snap-shot phase calibration:

- Rank $r_{p}=M^{2} / 4-1$;

- $r_{p}$ is $M / 2+1$ less than the number of unknowns.

2) Snap-shot amplitude calibration:

- Rank $r_{a}=M^{2} / 4$;

- $r_{a}$ is $M / 2$ less than the number of unknowns.

Although the system of calibration equations is always an over-determined system (for $M \geq 6$ ), the rank of the system of calibration equations for both cases is far smaller than the number of unknowns, which is infeasible for actual calibration.

\section{B. Rotational Sampling Measurement}

One of the most important advantages of the equispaced circular array is that the baselines with identical length can lead to fully redundant measurements when array rotation is considered. Here we use the assumptions that the variations of brightness temperature are negligible during a calibration period, and antenna elements are circularly polarized (the polarization will be discussed at the end of this section). The only condition that needs to be satisfied by array rotation is that the positions of antenna elements should overlap for each snap-shot measurement.

\section{1) Half revolution of array rotation:}

Fig. 4 shows the mentioned redundant measurements accomplished by a half revolution of array rotation, where the array is rotated counter-clockwise. Snapshots for each array rotation are shown in columns, while the baselines in different same-length baseline groups are shown in rows (diagonal baselines are not included).
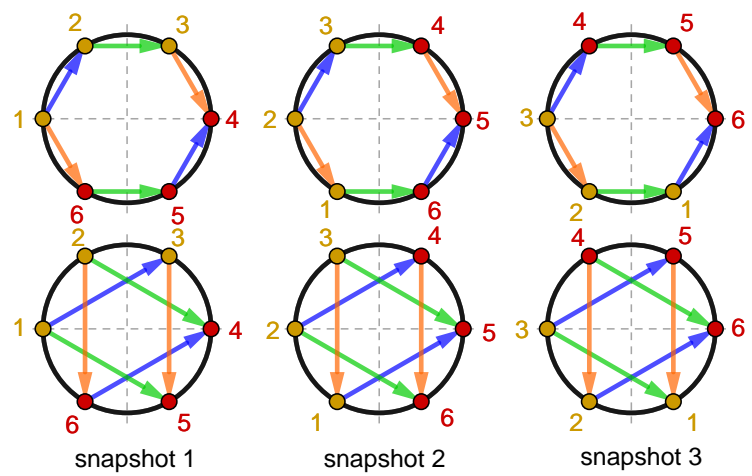

Fig. 4. Redundant measurements accomplished by a half revolution of array rotation.

It is obvious that every baseline conducts the measurements of all possible visibilities $(=M / 2$, also equals to the snapshots needed) during a half revolution of array rotation (e.g., baseline formed by $1^{\text {st }}$ and $2^{\text {nd }}$ element measures all three different colors of visibilities in the three snapshots during array rotation). Since there are $M$ baselines in each group of same-length baselines, the redundancy of all measured visibilities is increased from 2 to $M$. When building the system of calibration equations, each group of same-length baselines can be attributed to the same ideal visibility term, because all baselines belonging to the group have accomplished fully redundant measurements. Therefore, the total number of ideal visibility terms in the system of equations can be reduced to the number of same-length baseline groups, which is $M / 2-1$. Meanwhile, the number of separable factors remains $M$, so the number of unknowns reduces to $3 M / 2-1$, and the number of equations remains $M(M-2) / 2$.

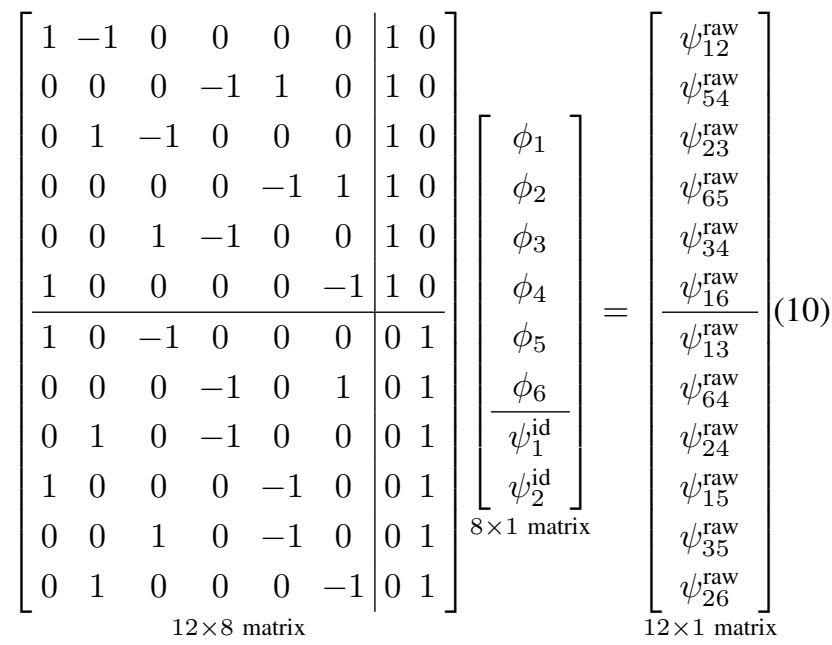

A specific structure of the system of calibration equations built by the half-revolution array rotation for phase calibration is expressed in (10) (constructed by the blue baselines in Fig. 4). As each same-length baseline group is attributed to the same ideal visibility phase by the array rotation, $\psi_{1}^{\text {id }}$ and $\psi_{2}^{\text {id }}$ in (10) are the phases of the ideal visibilities measured by these redundant baselines, and the subindex indicates their samelength baseline groups. The same-length baselines groups can also be identified by the relative separation of the index of every two separable phase factors.

Since the amplitude measurement does not depend on the $180^{\circ}$ rotation of the baseline, one can simply substitute the -1 coefficients by +1 to generate the coefficient matrix for amplitude calibration.

The corresponding relationship between coefficient matrix rank and number of unknowns can be summarized as follows:

1) Half-rotation phase calibration:

- Rank $r_{p}^{\prime}=3 M / 2-2$;

- $r_{p}^{\prime}$ is only one less than the number of unknowns;

2) Half-rotation amplitude calibration:

- Rank $r_{a}^{\prime}=3 M / 2-2$;

- $r_{a}^{\prime}$ is only one less than the number of unknowns.

The degree of freedom for both phase and amplitude calibration reduces to 1 , making redundant space calibration feasible.

Note that every baseline leads to fully redundant measurements of $M / 2$ visibilities during the half-revolution array 
rotation, and only one visibility for each group of samelength baselines is used in building the system of calibration equations (Equation (10) is built by only the blue visibility in Fig. 4). Hence, there are $M / 2$ systems of calibration equations to be built, and each solution of them represents an estimation of the system parameters. The redundant solutions to each system of calibration equations can be averaged to alleviate the influence of thermal noise, which is critical to improve the calibration performance and stability.

2) Complete revolution of array rotation:

As the aforementioned procedure of building system of equations can also be applied to the measurements with a complete array rotation, the features of array rotation can be further exploited. Measurements of a single baseline during a complete array rotation are shown in Fig. 5, where each subplot indicates an instantaneous measurement during the array rotation. Due to the centro-symmetric structure of equispaced circular array, each baseline measures all visibilities available in one snapshot by a complete revolution of array rotation $^{2}$. In other words, each baseline in the same-length baseline group measures all possible visibilities belonging to its group. Therefore, the averaging of all measurements of each baseline in this group can be attributed to the same averaged ideal visibility term.

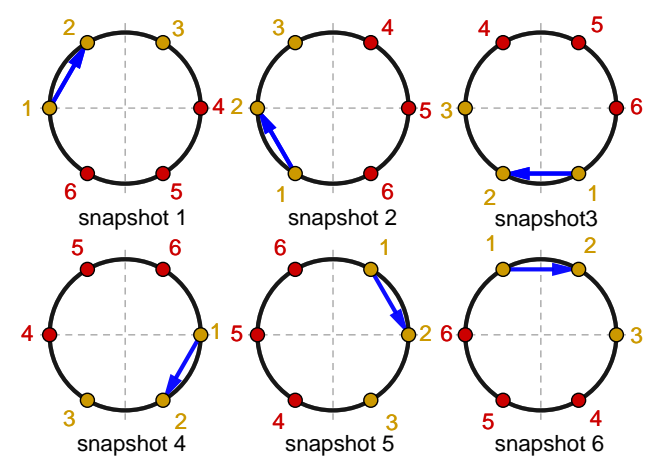

Fig. 5. Measurements of a baseline accomplished by a complete revolution of array rotation.

The averaging of a baseline's measurements during a complete revolution of array rotation can be described as

$$
\frac{1}{M} \sum_{p=1}^{M} V_{k l}^{\mathrm{raw}, p}=\gamma_{k} \cdot \gamma_{l}^{*} \cdot \frac{1}{M} \sum_{p=1}^{M} V_{k l}^{\mathrm{id}, p}+\frac{1}{M} \sum_{p=1}^{M} n_{k l}^{c, p}
$$

where the superscript $p$ denotes the index of snap-shot measurement, and it can be simplified into a general form as presented in (4)

$$
\overline{V_{k l}^{\mathrm{raw}}}=\gamma_{k} \cdot \gamma_{l}^{*} \cdot \overline{V_{k l}^{\mathrm{id}}}+\overline{n_{k l}^{c}}
$$

where $\overline{V_{k l}^{\text {raw }}}=\frac{1}{M} \sum_{p=1}^{M} V_{k l}^{\text {raw, } p}$ denotes the averaging of the measured complex correlations of the baseline $k-l, \overline{V_{k l}^{\text {id }}}=$

\footnotetext{
${ }^{2}$ For instance, baseline 1-2 measures the visibilities measured by 1-2, 6-1, $5-6,4-5,3-4$, and $2-3$ in snapshot 1 , by the consecutive snapshots during array rotation.
}

$\frac{1}{M} \sum_{p=1}^{M} V_{k l}^{\mathrm{id}, p}$ denotes the averaged ideal visibility term, and $\overline{n_{k l}^{c}}=\frac{1}{M} \sum_{p=1}^{M} n_{\mathrm{c}}^{p}$ denotes the averaged noise term.

Under the assumption that variations of the observed scene are negligible during the array rotation (which requires a relatively short rotation period), the averaged ideal visibility term $\overline{V_{k l}^{\mathrm{id}}}$ is identical for all baselines in the same-length baseline group. It allows that the averaged measurements of complex correlations can be used as the constant terms in the right side of system of equations, while the coefficient matrix for phase and amplitude calibration remains the same structure. Therefore, the relationship between the rank of coefficient matrix and the number of unknowns in the half revolution of array rotation still works on the complete revolution of array rotation:

1) For complete-rotation phase calibration:

- Rank $r_{p}^{\prime \prime}=3 M / 2-2$;

- $r_{p}^{\prime \prime}$ is only one less than the number of unknowns;

2) For complete-rotation amplitude calibration:

- Rank $r_{a}^{\prime \prime}=3 M / 2-2$;

- $r_{a}^{\prime \prime}$ is only one less than the number of unknowns.

The improvements of the calibration scheme accomplished by a complete revolution of array rotation come from two parts:

1) The noise term is averaged for $M$ times, so that the noise level can be decreased by a factor of $M$ (for noise variance);

2) All measurements of the complex correlations are used in a single system of equations, and only a single solving process is needed, which significantly reduces the time consumption of the solving process.

As a quick review of the aforementioned analysis, different ways to build the system of calibration equations for both phase and amplitude calibration are summarized as follows:

1) Half revolution of array rotation:

\section{A) Phase calibration}

i) Build $M / 2$ systems of calibration equations, then average the $M / 2$ solutions.

1.B) Amplitude calibration

i) Build $M / 2$ systems of calibration equations, then average the $M / 2$ solutions;

ii) Average the $M / 2$ measured amplitudes of complex correlations for each baseline, then build one single system of calibration equations;

iii) Average the $M / 2$ natural logarithm of measured amplitudes of complex correlations for each baseline, then build one single system of calibration equations.

2) Complete revolution of array rotation

2.A) Phase calibration

i) Build $M$ systems of calibration equations, then average the $M$ solutions;

ii) Average the $M$ measured complex correlations for each baseline, then use the phases of averaged complex correlations to build one single system of calibration equations.

2.B) Amplitude calibration 
i) Build $M$ systems of calibration equations, then average the $M$ solutions;

ii) Average the $M$ measured complex correlations for each baseline, then build one single system of calibration equations;

iii) Average the $M$ amplitudes of the measured complex correlations for each baseline, then build one system of calibration equations;

iv) Average the $M$ natural logarithm of measured amplitudes of complex correlations for each baseline, then build one system of calibration equations.

The main difference between phase and amplitude calibration is that the measured phases cannot be averaged, no matter in which array rotation scheme. It is due to the measured phase is naturally wrapped in the principle interval of $2 \pi$, and averaging of the measured phases does not always ensure the consistency between different baselines. By comparison, amplitude calibration does not suffer from the wrapping problem, and the measured amplitude remains identical when a $180^{\circ}$ rotation happens on the baseline. So the averaging operation for amplitude calibration can be conducted either on the amplitude level or on the natural logarithm of amplitude level. Overall, the approach of averaging before solving process is a better choice because it significantly reduces the time consumption of calibration. Thus, a complete revolution of array rotation is especially preferable for phase calibration. On the other hand, amplitude calibration is less influenced by the specific averaging methods, but the calibration performance still differs due to its special noise characteristic [54].

The rotational sampling calibration strategy also works on the equispaced circular array with an odd number of elements. However, the fully redundant measurements of each visibility can only be accomplished by a complete revolution of array rotation, since the array with an odd number of elements does not have any instantaneous redundancy in a single snapshot. Also considering the inherent side effects brought by the array with an odd number of elements, the array with an even number of elements is more favorable.

Circular polarization perfectly fits the proposed calibration strategy, since the array rotation will not introduce any difference on the observed brightness temperature of each rotated snapshot $^{3}$. The proposed calibration strategy does not require circular-polarized antenna elements compulsively, but it should be pointed out that if linear polarization is employed, the linear-polarized brightness temperature being observed varies during array rotation. However, the calibration strategy can still be implemented if all antenna elements' polarizations are unified to the same direction by careful installation.

\section{Principle of Phase Retrieval Technique}

The rotational sampling strategy permits the phase retrieval from the corresponding system of calibration equations as described in Section III-B. Several techniques have been explored to solve the phase retrieval problem, such as the leastsquares method [33] and iterative method [55]. However, the

\footnotetext{
${ }^{3}$ The wind-up effect of circular polarization cancels out because all antenna elements rotate along with the array rotation.
}

phase wrapping puzzle has been reported to be unavoidable when trying to retrieve the unknown phase from the system of calibration equations [27], [35]. This trouble comes from the measurement of phase, which is achieved by measuring the real and imaginary part of a phasor, then calculating the arctangent function of the ratio between them. The phase measured by this way is naturally wrapped into the primary interval $[-\pi, \pi$ ), and the original phase (may not be in the primary interval) is lost and cannot be recovered. The relationship between the original phase and measured phase can be described as

$$
\begin{aligned}
\psi_{k l}^{\text {measured }} & =\arctan 2\left\{\operatorname{Im}\left(V_{k l}^{\text {raw }}\right), \operatorname{Re}\left(V_{k l}^{\text {raw }}\right)\right\} \\
& =\operatorname{Mod}\left\{\psi_{k l}^{\text {original }}\right\} \\
& =\psi_{k l}^{\text {original }}+2 k \pi, \quad k=0, \pm 1, \pm 2 \cdots
\end{aligned}
$$

where the function $\operatorname{Mod}[\cdot]$ returns the wrapped phase in the primary interval $[-\pi, \pi)$. One can only preserve the correctness of the solution when the original phases exactly fall into the primary interval ( $k=0$ for every measured phase), which is unrealistic in practical case.

The phase wrapping puzzle inherently exists in the phase retrieval problem. Fortunately, the unique structure of the system of phase calibration equations established by array rotation provides an ingenious approach to deal with it.

\section{A. Property of the Fixed $\pi$-ambiguity}

As described in Section III-B, baselines with identical length can perform fully redundant measurements by array rotation. The ideal visibility terms in the calibration equations can be attributed to the same ones for all baselines with identical length. Assuming that $N=M / 2$ is half the number of elements, and $d$ is the index of the ideal phase term (the value of $d$ also equals to the sequential index of the samelength baseline group), the phase calibration equations can be summarized as follows:

$$
\begin{array}{r}
\left\{\begin{aligned}
\phi_{i}-\phi_{i+d}+\psi_{d}^{\text {id }}=\psi_{m_{1}}^{\text {raw }}, & 1 \leq i \leq N-d \\
\phi_{i+d+N}-\phi_{i+N}+\psi_{d}^{\text {id }}=\psi_{m_{2}}^{\text {raw }}, & 1 \leq i \leq N-d
\end{aligned}\right. \\
\left\{\begin{array}{cl}
\phi_{i}-\phi_{i+d}+\psi_{d}^{\text {id }}=\psi_{n_{1}}^{\text {raw }}, & N-d<i \leq N \\
\phi_{i+d-N}-\phi_{i+N}+\psi_{d}^{\text {id }}=\psi_{n_{2}}^{\text {raw }}, & N-d<i \leq N
\end{array}\right.
\end{array}
$$

where $\phi_{i}$ denotes the separable phase factor of $i^{t h}$ element, $\psi_{d}^{\text {id }}$ denotes the ideal phase of the visibility in the current group of same-length baselines; and $\psi_{x}^{\text {raw }}$ is used to indicate the measured phase, the subscript of it can be inferred by the antenna pair forming the corresponding equation. The index $d$ is $1 \leq d \leq N-1$, when the longest baselines have been excluded. The separation of (14) and (15) is caused by the circular shift of element indexing. Note that the measured phase is wrapped into the primary interval $[-\pi, \pi)$, while the original phase (right-hand side of the equations) is lost. What we can do is to estimate $\phi_{i}$ and $\psi_{d}^{\text {id }}$ by $\hat{\phi}_{i}$ and $\hat{\psi}_{d}^{\text {id }}$, with the given measured phases.

It can be proved that (see Appendix for details) the solution of the system of phase calibration equations (14) and (15) has 
a unique property called the fixed $\pi$-ambiguity, which means that the estimations of the separable phase factors $\hat{\phi}_{i}$ and the ideal phase terms $\hat{\psi}_{d}^{\text {id }}$ are different from their actual values by two possible arithmetic sequences, with the same common difference of either 0 or $\pi$. Also, there are two unsolvable constant phase biases for the separable phase factors and the ideal visibility phases, due to the one degree of freedom of the system of phase calibration equations. The constant phase bias for the separable phase factors can be simply ignored because the bias cancels out when the phase difference between any two receiving channels is calculated (i.e., the baseline's phase error). On the other hand, the constant phase bias for the ideal visibility phases has been proved (see Appendix) to be identical to the common difference in the mentioned arithmetic sequences; in other words, either 0 or $\pi$.

The fixed $\pi$-ambiguity provides two possible solutions to the system of phase calibration equations, which can be described as

$$
\begin{aligned}
& \left\{\begin{array}{l}
\hat{\phi}_{i}^{\text {set } 1}=\hat{\phi}_{i}, \quad i=1,2, \ldots, M \\
\hat{\psi}_{d}^{\text {set } 1}=\hat{\psi}_{d}^{\mathrm{id}}, \quad d=1,2, \ldots, P
\end{array}\right. \\
& \begin{cases}\hat{\phi}_{i}^{\mathrm{set} 2}=\hat{\phi}_{i}+i \cdot \pi, & i=1,2, \ldots, M \\
\hat{\psi}_{d}^{\mathrm{set} 2}=\hat{\psi}_{d}^{\mathrm{id}}+d \cdot \pi, & d=1,2, \ldots, P\end{cases}
\end{aligned}
$$

where $\hat{\phi}_{i}$ and $\hat{\psi}_{d}^{\text {id }}$ are the raw estimations of separable phase factors and ideal visibility phases, respectively; and $P$ equals the number of same-length baseline groups used in the system of phase calibration equations. The case with the common difference of 0 is denoted by superscript "set 1 ", and the case with the common difference of $\pi$ is denoted by superscript "set $2 "$.

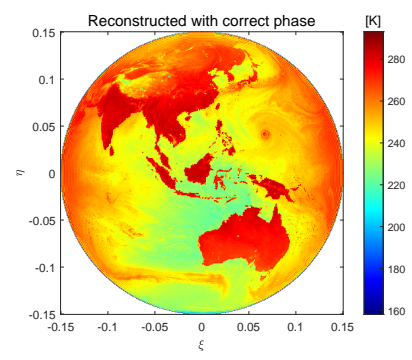

(a)

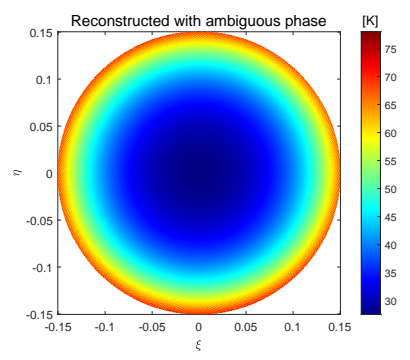

(b)
Fig. 6. Reconstructed brightness temperature with the fixed $\pi$-ambiguity: (a) correct phase, (b) ambiguous phase.

This kind of ambiguity can be easily eliminated by a single image reconstruction. Fig. 6 shows the different representations of the two possible phase solutions on the reconstructed brightness temperature image, where the correctness of two possible solutions can be determined by visual inspection (the image reconstruction technique for polar sampling grid can be found in [48]). Since the separable phase factor of receiving channel does not change rapidly over time, the unambiguous solution inferred by the image reconstruction can be used as a priori information to eliminate the fixed $\pi$-ambiguity in the subsequent phase calibration process.

\section{B. Solving Techniques}

However, the overdetermined system of phase calibration equations still cannot be solved by the ordinary least-squares method or Moore-Penrose inverse [56], because the overdetermined system with wrapped phase measurements is potentially inconsistent. It can be verified by comparing the rank of coefficient matrix and the rank of its augmented matrix, the system of equations is consistent if and only if the two ranks are equal (Rouché-Capelli theorem [57]). The system of equations is consistent when the phase measurements (constant terms) are unwrapped because they are linear combinations of the unknowns to be solved. If the phase measurements are wrapped, the system of equations potentially becomes inconsistent, and one will obtain some contradictory equations after Gaussian elimination. Another explanation for it is that any division involved in the solving procedure will introduce phase ambiguity (not only $\pi$-ambiguity), since the wrapped phases are different from their true values by $\pm 2 k \pi$ ( $k$ is a positive integer). As a result, the solutions obtained by the ordinary least-squares method and inversion-related methods are most probably incorrect, even in noise-free cases. They can only be used when the separable phase factors and the ideal visibility phases are small enough (close to zero), to avoid phase wrapping occurring in the phase measurement, which is not realistic in general case. Otherwise, the phase unwrapping technique for either the measured phases or the retrieved phases is required unavoidably.

An alternative methodology for phase retrieval is to fit complex phasors rather than the measured phases [33], which can be achieved by minimizing the objective function defined as

$$
\mathcal{F}=\sum_{k, l}^{M} w_{k l}\left\{\left[\operatorname{Re}\left(\Delta_{k l}\right)\right]^{2}+\left[\operatorname{Im}\left(\Delta_{k l}\right)\right]^{2}\right\}
$$

where $w_{k l}$ denotes the weighting function of the baselines, and the quantity $\Delta_{k l}$ indicates the misfit between the measured complex correlation's phasor with a unity amplitude and the corresponding model phasor

$$
\Delta_{k l}=\exp \left(i \cdot \psi_{k l}^{\mathrm{raw}}\right)-\exp \left[i \cdot\left(\phi_{k}-\phi_{l}+\psi_{k l}^{\mathrm{id}}\right)\right] .
$$

The objective function can be further simplified by the properties of trigonometric functions

$$
\mathcal{F}=\sum_{k, l}^{M} w_{k l} \cdot \sin ^{2}\left[\frac{\psi_{k l}^{\mathrm{raw}}-\left(\phi_{k}-\phi_{l}+\psi_{k l}^{\mathrm{id}}\right)}{2}\right] .
$$

Although the fit of complex phasors successfully removes the $2 \pi$ ambiguity of the measured phase, it also should be noted that the objective function can be approaching zero from either $0^{\circ}$ direction or $180^{\circ}$ direction in the complex plane. It agrees with the fact that $\psi_{k l}^{\text {raw }}-\left(\phi_{k}-\phi_{l}+\psi_{k l}^{\text {id }}\right)$ can be either 0 or $2 \pi$ when the objective function in (20) is being minimized to approach zero. Fortunately, the misfit does not need any extra correction because it has already been degenerated into the fixed $\pi$-ambiguity due to the unique structure of the system of phase calibration equations. The residual misfit is expressed 
by the wrong solution of the two possible solutions inferred by the fixed $\pi$-ambiguity.

The minimization of the objective function expressed in (20) is a nonlinear least-squares problem, which can be effectively solved by sophisticated iterative methods, such as Trust-region Reflective Algorithm [58], [59]. The Jacobian matrix can be analytically calculated based on the defined objective function, which reduces the time consumption of each iteration. Apart from that, there are two essential quantities to be defined before the minimization algorithm begins, which are: 1) the weighting function and 2) the initial guess of the solution. The weighting function should be carefully designed based on the behavior of noise term. The basic idea is to define $w_{k l}=1 / \sigma_{k l}$ when the noise obeys a zero-mean Gaussian distribution (where $\sigma_{k l}$ is the standard deviation of the corresponding Gaussian noise). The detailed noise analysis is the subject of a companion paper [54]. Only the selection of initial guess is discussed here.

\section{Selection of Initial Guess}

The initial guess is of great importance for iterative optimization algorithms, especially under noise conditions. The optimization solvers typically find a local minimum because the gradient-related methods are commonly used to update the solution in each iteration. One can only expect the global minimum is found when the initial guess is provided in the basin of attraction of a global minimum. Although some global optimization techniques have been proven to be effective, such as Simulated Annealing (SA) [60] and Genetic Algorithms (GA) [61], it has to be pointed out that they are timeconsuming especially dealing with a large-scale problem. The best way to ensure the success of phase calibration is to provide a good-enough initial guess, which leads to a reasonable solution.

As it has been explained previously, the phase wrapping problem occurs when the system of phase calibration equations is inconsistent. Although it is the case that happened in most of the phase-retrieval problems, we have found that the solution obtained by matrix inversion can still be reasonable under some specific circumstances. The core idea is to make the system of phase calibration equations consistent by some basic matrix operations, even with the originally measured (wrapped) phases as constant terms.

The inconsistency of the system of phase calibration equations results from the wrapped constant terms, which will result in contradictory equations after row reductions. So the first criterion we apply is to reduce the number of equations by removing equations from the system of phase calibration equations as much as possible, in order to avoid potential contradictions. The second criterion is to find an indicator to preserve the degree of freedom of the system of equations, which is naturally the rank of the coefficient matrix.

After equation removal, the information contained in the original system of equations is reduced significantly, and the solution becomes much more sensitive to noise. Based on the inherent property of Fourier Transform of the natural scene, the energy in the frequency domain (spatial frequency in our case) is concentrated in the low-frequency part, so the baselines with shorter baseline length have a higher signal-to-noise ratio. Following the derivations presented in the Appendix, it can be verified that the fixed $\pi$-ambiguity still holds when only the first two same-length baseline groups are used to build the system of phase calibration equations $(d=1$ and 2$)$, and there are two degrees of freedom as well. Thus, the two shortest baselines are selected to form the system of equations before equation removal begins.

Without loss of generality, the phase of the first element is set to zero $\left(\phi_{1}=0\right)$ because the constant phase bias in the separable phase factors is irrelevant when the baseline's phase error is calculated. Consequently, the first unknown, and also the entire first column of coefficient matrix can be deleted, and the system of equations becomes full-rank before equation removal starts.

The equation removal is conducted based on the idea of recursive backtracking, and the detailed steps are summarized as follows:

1) Build the system of phase calibration equations with the first two groups of same-length baselines, initialize $i=1$;

2) Record the current system of equations as subset $B_{i}$, initialize $e_{i}=1$, where $e_{i}$ denotes the index of equation to be removed in this loop;

3) Remove equation $e_{i}$ from the system of equations $B_{i}$;

4) Check the rank of coefficient matrix:

- If the rank does not change, set $i=i+1$, continue from step 2);

- If the rank is reduced, preserve equation $e_{i}$, set $e_{i}=$ $e_{i}+1$ and get back to step 3);

5) If all equations have been checked, but the rank cannot be preserved, return to the subset $B_{i-1}$, set $e_{i-1}=e_{i-1}+1$, and $i=i-1$, continue from step 3 );

6) Stop until the coefficient matrix becomes a square matrix.

Once the coefficient matrix becomes a square matrix, the system of phase calibration equations can be solved by simple matrix inversion. A typical full-rank square coefficient matrix is (originated from the coefficient matrix shown in (10))

$$
A=\left[\begin{array}{rrrrr|rr}
0 & 0 & 0 & -1 & 1 & 1 & 0 \\
0 & 0 & 0 & 0 & -1 & 1 & 0 \\
0 & 0 & -1 & 0 & 1 & 0 & 1 \\
1 & 0 & -1 & 0 & 0 & 0 & 1 \\
0 & 0 & 0 & -1 & 0 & 0 & 1 \\
0 & 1 & 0 & -1 & 0 & 0 & 1 \\
1 & 0 & 0 & 0 & -1 & 0 & 1
\end{array}\right]
$$

where the vertical line indicates the boundary between two kinds of matrix entries that corresponding to the separable phase factors and the ideal visibility phases, respectively.

On the other hand, the inverse of (21) is shown at the top of next page, where the horizontal line also indicates the boundary between two kinds of matrix entries that corresponding to the separable phase factors and the ideal visibility phases, respectively. 


$$
A^{-1}=\left[\begin{array}{rrrrrrr}
\frac{1}{2} & -\frac{1}{2} & -\frac{1}{2} & \frac{1}{2} & -\frac{1}{2} & 0 & \frac{1}{2} \\
0 & 0 & 0 & 0 & -1 & 1 & 0 \\
\frac{1}{2} & -\frac{1}{2} & \frac{1}{2} & -\frac{3}{2} & -\frac{1}{2} & 0 & \frac{3}{2} \\
0 & 0 & 1 & -1 & -1 & 0 & 1 \\
\frac{1}{2} & -\frac{1}{2} & \frac{1}{2} & -\frac{1}{2} & -\frac{1}{2} & 0 & \frac{1}{2} \\
\hline \frac{1}{2} & \frac{1}{2} & \frac{1}{2} & -\frac{1}{2} & -\frac{1}{2} & 0 & \frac{1}{2} \\
0 & 0 & 1 & -1 & 0 & 0 & 1
\end{array}\right]
$$

It can be noticed that the inverse of the coefficient matrix has a unique structure. Apart from integers, only fractions with the denominator of 2 appears in the matrix entries. The solution obtained by the corresponding square matrix inversion can be interpreted as follows:

1) The divided-by-2 operation (the denominator of 2) will only generate $\pi$-ambiguity. Recalling that the solution of the system of equations will be calculated by rightmultiplying $A^{-1}$ by the constant terms (wrapped phase measurements): if the wrapped phase is different from the original value with an odd $k$ in $2 k \pi$, the $\pi$-ambiguity happens; and if $k$ is an even number, it does not make any mistake;

2) From the column's perspective, the divided-by- 2 operations act on the same constant terms (for every row in (22), if the divided-by-2 operations exist, they appear consistently in columns 1-5 and 7), which implies the solution influenced by the potential ambiguity will have the consistent $\pi$-ambiguity (whether ambiguous or not, simultaneously);

3) From the row's perspective, the divided-by-2 operations appear every two rows (remember the boundary between separable phase factors and ideal visibility phases), which implies that every two solved separable factors and solved ideal phases will be influenced by the potential ambiguity.

Overall, the solution obtained by square matrix inversion agrees with the property of the fixed $\pi$-ambiguity inferred by the original system of phase calibration equations. Here we define the initial guess obtained by the square matrix inversion as the first initial guess, which is denoted by

$$
\mathbf{x}_{1}^{\mathrm{ini}}=\left\{\overline{\phi_{1}}, \overline{\phi_{2}}, \ldots, \overline{\phi_{M}} \mid \overline{\psi_{1}^{\text {id }}}, \overline{\psi_{2}^{\text {id }}}\right\}
$$

where $\overline{\phi_{1}}=0$. Note that only two ideal visibility phases are contained in the first initial guess, since the baselines in the first two groups of same-length baselines are used to estimate the first initial guess.

The residual error in the first initial guess will be significant because very few measurements are utilized. The quality of the first initial guess can be improved by minimizing the objective function in (20), with all baseline measurements in the two corresponding same-length baseline groups, provided the first initial guess as a starting point. The solution is defined as the second initial guess

$$
\mathbf{x}_{2}^{\text {ini }}=\left\{\overline{\overline{\phi_{1}}}, \overline{\overline{\phi_{2}}}, \ldots, \overline{\overline{\phi_{M}}} \mid \overline{\overline{\psi_{1}^{\text {id }}}}, \overline{\overline{\psi_{2}^{\text {id }}}}\right\} .
$$

To ensure the best phase calibration performance, all available baseline measurements should be used in constructing the system of phase calibration equations (but with different weights). So the initial guess of ideal visibility phases needs to be further extended for the baselines in the other same-length baseline groups. The estimation of ideal visibility phases in the other same-length baseline groups can be attained by the phase calibration model in (5) and the redundant measurements of each ideal visibility terms:

$$
\overline{\overline{\overline{\psi_{d}^{\mathrm{id}}}}}=\arg \left\{\frac{\sum_{\{k l\} \in S_{d}} \exp \left[i \cdot\left(\psi_{k l}^{\mathrm{raw}}-\overline{\overline{\phi_{k}}}+\overline{\overline{\phi_{l}}}\right)\right]}{M}\right\}
$$

where the average is calculated at complex phasor's domain to avoid any potential anomaly caused by the arithmetic mean of the phases. The denominator is equal to the total number of elements $M$ since there are $M$ baselines in each same-length baseline group and each baseline realizes the measurement of the same visibility.

So far, initial guesses for all possible unknowns involved in the phase calibration equations are reasonably obtained, which is defined as the third initial guess

$$
\mathbf{x}_{3}^{\text {ini }}=\left\{\overline{\overline{\phi_{1}}}, \overline{\overline{\phi_{2}}}, \ldots, \overline{\overline{\phi_{M}}} \overline{\overline{\overline{\psi_{1}^{\text {id }}}}}, \overline{\overline{\overline{\psi_{2}^{\text {id }}}}}, \ldots, \overline{\overline{\overline{\psi_{d}^{\text {id }}}}}\right\} .
$$

Although the residual phase error in the third initial guess is amplified along with the relative distance between every two elements, which agrees with the conclusion shown in [37]. It still works as a good-enough initial guess to converge to a reasonable solution [54].

\section{Principle of Amplitude Retrieval technique}

Compared to the phase retrieval, amplitude retrieval is much simpler because it does not need to deal with any "amplitude wrapping" problem. The original amplitude calibration model is described by products that cannot be used to build a linear system of amplitude calibration equations. A proper way is to linearize the equations by taking the natural logarithm of both sides of (6), as shown in (7) [37].

Since the degree of freedom of the system of amplitude calibration equations has been reduced to 1 by applying array rotation, it is natural to set $\hat{g}_{1}=1$ as the reference amplitude $\left(\ln \left(\hat{g}_{1}\right)=0\right)$. The first unknown, and also the entire first column of coefficient matrix can be deleted. Hence, the overdetermined system of amplitude calibration equations becomes full-rank, it can be solved by the weighted leastsquare method.

$$
\hat{\mathbf{y}}=\left(B^{\top} W_{A} B\right)^{-1} B^{\top} W_{A} \mathbf{d}
$$

where $B$ is the coefficient matrix for amplitude calibration, $W_{A}$ is the weighing matrix, $\mathbf{d}$ is the natural logarithm of the measured amplitudes of complex correlations, and $(\cdot)^{\top}$ denotes the matrix transpose. Noted that the weighing matrix 
should be carefully designed based on the noise's behavior, especially when the natural logarithm of the measured amplitude is calculated to conduct unknown's linearization [54].

As $\hat{g}_{1}$ is set to 1 to establish a full-rank coefficient matrix, the unknowns solved by weighted least-square method are different from their true values by a multiplicative factor related to the true value of $g_{1}$

$$
\begin{gathered}
g_{k}=\hat{g}_{k} \cdot g_{1}, \quad k=2,3, \ldots, M \\
\left|V_{d}^{\mathrm{id}}\right|=\left|\hat{V_{d}^{\mathrm{id}}}\right| / g_{1}^{2}, \quad d=1,2, \ldots, P
\end{gathered}
$$

where $\hat{g}_{k}$ and $\left|\hat{V}_{d}^{\text {id }}\right|$ are the raw estimations of the separable amplitude factors and the ideal visibility amplitudes, respectively, they are consistent with the assumption $\hat{g}_{1}=1$; and $P$ denotes how many groups of same-length baselines are used in building the system of amplitude calibration equations.

The amplitude calibration conducted with one degree of freedom is a relative calibration, which calibrates the relative consistency of the amplitudes of each measured visibility. There is still a free variable needed to be determined by the other kinds of auxiliary calibration methods, such as the classical noise injection. As a priori brightness temperature of the observed scene can be provided by the well-modeled brightness temperature or the actual measurements of the real aperture radiometers on polar-orbiting satellites, it offers a potential approach to conduct absolute amplitude calibration at the brightness temperature level [54], in association with the proposed method.

\section{CONCLUSION}

In this paper, a novel calibration strategy for both phase and amplitude calibration based on redundant baseline measurements has been proposed. The fully redundant measurements for each visibility accomplished by the unique structure of the equispaced circular array and rotational sampling strategy permit the calibration with minimal aid of any auxiliary method. It further suggests the layout of elements that a rotating circular array should have in order to ease its calibration.

It has been analytically proven that the phase calibration carried out by this strategy has a unique property of the fixed $\pi$-ambiguity, and this kind of ambiguity can be easily eliminated by a single image reconstruction. It successfully solves the inevitable problem of phase wrapping encountered in the phase retrieval of redundant space calibration, and no other phase unwrapping technique is needed. The residual degree of freedom cancels out when the baseline phase is calculated, so the phase calibration is well-defined and selfcontained. On the other hand, the amplitude calibration acts as a relative calibration which calibrates the relative consistency of the amplitudes of each measured visibility.

This study provides a potential scheme of interferometric synthetic aperture radiometer design for geostationary passive microwave observations, especially as the calibration is difficult to be implemented by the conventional noise injection method. The simulation results and discussion of the proposed calibration strategy are left for a later accompanying paper.
It should be pointed out that the visibility sampled during array rotation is an averaged version of the visibility function along its sampling trajectory. It can be recognized as the "blurring effect" of visibility sample when a large integration time (slow array rotation) is applied to the snap-shot measurement. On the other hand, fast array rotation unavoidably induces a structural deformation of the array, and the visibility sampling position will differ from its nominal value accordingly. A dedicated analysis needs to be accomplished in a future study considering the actual implementation of the proposed calibration strategy.

\section{APPENDIX}

\section{PROOF OF THE FIXED $\pi$-AMBIGUITY}

As described in Section IV-A, baselines with identical length lead to fully redundant measurements of all possible visibilities by array rotation. Then, the ideal visibility terms in the calibration equations can be referred to the same ones for all baselines with identical length. It is assumed that there is a set of estimation of separable phase factors $\hat{\phi}_{i}$ and ideal visibility phases $\hat{\psi}_{d}^{\text {id }}$, which satisfy (14) and (15), but with arbitrary phase wrapping factors. This assumption is compatible with the use of the measured (wrapped) phase as a constant term in the phase calibration equations. Using the baseline indexing as illustrated in Fig. 4 (blue baselines), the phase calibration equations in (14) and (15) can be rewritten with $\hat{\phi}_{i}$ and $\hat{\psi}_{d}^{\text {id }}$

$$
\begin{aligned}
& \left\{\begin{aligned}
\hat{\phi}_{i}-\hat{\phi}_{i+d}+\hat{\psi}_{d}^{\mathrm{id}}=\hat{\psi}_{m_{1}}^{\mathrm{raw}}, & 1 \leq i \leq N-d \\
\hat{\phi}_{i+d+N}-\hat{\phi}_{i+N}+\hat{\psi}_{d}^{\mathrm{id}}=\hat{\psi}_{m_{2}}^{\mathrm{raw}}, & 1 \leq i \leq N-d
\end{aligned}\right. \\
& \left\{\begin{array}{r}
\hat{\phi}_{i}-\hat{\phi}_{i+d}+\hat{\psi}_{d}^{\text {id }}=\hat{\psi}_{n_{1}}^{\text {raw }}, \quad N-d<i \leq N \\
\hat{\phi}_{i+d-N}-\hat{\phi}_{i+N}+\hat{\psi}_{d}^{\text {id }}=\hat{\psi}_{n_{2}}^{\text {raw }}, \quad N-d<i \leq N
\end{array}\right.
\end{aligned}
$$

where terms $\hat{\psi}_{x}^{\text {raw }}=\psi_{x}^{\text {raw }}+2 k_{x} \pi$ denote the phase wrapping caused by the phase measurement and inexact estimations of $\phi_{i}$ and $\psi_{d}^{\mathrm{id}}, k_{x}$ is an integer; and the subscript of $\hat{\psi}_{x}^{\text {raw }}$, $\psi_{x}^{\text {raw }}$ and $k_{x}$ can be inferred by the antenna pair forming the corresponding equation. The index $d$ is $1 \leq d \leq N-1$, when the longest baselines are excluded.

Due to the $2 \pi$ periodicity of the phase, the system of equations defined by (14) and (15) has infinite solutions. Defining the difference between the true unknowns $\phi_{i}, \psi_{d}^{\text {id }}$ and the estimation set of them $\hat{\phi}_{i}, \hat{\psi}_{d}^{\text {id }}$ as $S_{i}$ and $X_{d}$, respectively

$$
\left\{\begin{array}{cl}
S_{i}=\phi_{i}-\hat{\phi}_{i}, & i=1,2, \cdots, M \\
X_{d}=\psi_{d}^{\text {id }}-\hat{\psi}_{d}^{\text {id }}, & d=1,2, \cdots, N-1
\end{array}\right.
$$

where the possible values for $S_{i}$ and $X_{d}$ are not necessarily limited to the natural period of phase.

The following steps will demonstrate that the solution patterns inferred by (A-1) and (A-2) can be used to remove irregular phase ambiguity in the phase retrieval problem. 


\section{A. Solution Pattern Inferred by (A-1a) and (A-2a)}

Since the structures of (A-1a) and (A-2a) are identical, they can be rewritten as an unity by using the difference between the true unknowns and the estimation set defined by (A-3)

$$
S_{i}-S_{i+d}+X_{d}=2 k_{a} \pi, \quad\left\{\begin{array}{l}
i=1,2, \ldots, N \\
d=1,2, \ldots, N-1
\end{array}\right.
$$

where the $2 k_{a} \pi$ term is the phase wrapping factor as defined after (A-1a) and (A-2a), and the definitions of (14a) and (15a) are used in the derivation of (A-4). For simplicity, the subscript of $k_{a}$ is described as $a=\{i, d\}$ in the following derivation.

Expanding (A-4) by every possible $d$, we have

$$
\begin{cases}d=1: & S_{i}-S_{i+1}=-X_{1}+2 k_{i, 1} \pi \\ d=2: & S_{i}-S_{i+2}=-X_{2}+2 k_{i, 2} \pi\end{cases}
$$

where the $2 k_{i, d} \pi$ terms in each equation are not necessarily identical, as they vary with both $i$ and $d$.

Since $S_{i}$ and $X_{d}$ denote the difference between phases, the $2 k_{i, d} \pi$ terms in (A-5) can be neglected without loss of generality. Subtracting (A-5-1) from (A-5-2), (A-5-2) from (A$5-3), \cdots,(\mathrm{A}-5-\mathrm{N}-2)$ from $(\mathrm{A}-5-\mathrm{N}-1)$, then rewriting $(\mathrm{A}-5-1)$ in the first equation, we obtain

$$
\begin{cases}S_{i}-S_{i+1} & =-X_{1} \\ S_{i+1}-S_{i+2} & =X_{1}-X_{2} \\ S_{i+2}-S_{i+3} & =X_{2}-X_{3} \\ \quad \vdots & \vdots \\ S_{i+N-2}-S_{i+N-1} & =X_{N-2}-X_{N-1} .\end{cases}
$$

where the index $i$ is $1 \leq i \leq N$ for each equation in (A-6).

The equations in (A-6) indicate that every consecutive part of sequence $S_{i}$ is an arithmetic sequence with a common difference of the combination of $X_{d}$ terms (but not the complete sequence at this point $)^{4}$. On the other hand, every arithmetic sequence expressed by (A-6) shares at least two identical elements of $S_{i}$; thus, it can be deduced that their common differences are equal, which yields

$$
\left\{\begin{array}{l}
-X_{1}=X_{1}-X_{2} \\
-X_{1}=X_{2}-X_{3} \\
\vdots \\
-X_{1}=X_{N-2}-X_{N-1} .
\end{array}\right.
$$

From (A-7) it can be inferred that $X_{d}$ is an arithmetic sequence with a common difference of $X_{1}$

$$
X_{d}=X_{1}+X_{1}(d-1), \quad d=1,2, \cdots, N-1 .
$$

Combining (A-6) and (A-8), we have

$$
S_{i}=S_{1}+X_{1}(i-1), \quad i=1,2, \cdots, 2 N-1
$$

\footnotetext{
${ }^{4}$ For instance, considering an array with eight elements $(N=4)$, it yields the common difference of $\left\{S_{1}, S_{2}, S_{3}, S_{4}, S_{5}\right\}$ is $-X_{1}$, the common difference of $\left\{S_{2}, S_{3}, S_{4}, S_{5}, S_{6}\right\}$ is $X_{1}-X_{2}$, the common difference of $\left\{S_{3}, S_{4}, S_{5}, S_{6}, S_{7}\right\}$ is $X_{2}-X_{3}$.
}

where the common difference of $S_{i}$ is $X_{1}$.

Equation (A-8) and (A-9) indicate that the estimation set satisfying (A-1a) and (A-2a) has two degrees of freedom: a constant phase term $S_{1}$ and $X_{1}$ for the separable phase factors and the ideal visibility phases, respectively; and a phase slope term recognized by $X_{1}$.

\section{B. Solution Pattern Inferred by (A-1b) and (A-2b)}

Rewriting (A-1b) and (A-2b) using $S_{i}$ and $X_{d}$ defined in (A-3)

$$
\begin{aligned}
& S_{i+d+N}-S_{i+N}=-X_{d}+2 k_{b} \pi, \quad\left\{\begin{array}{l}
i=1,2, \ldots, N-d \\
d=1,2, \ldots, N-1
\end{array}\right. \\
& S_{i+d-N}-S_{i+N}=-X_{d}+2 k_{c} \pi, \quad\left\{\begin{array}{l}
N-d<i \leq N \\
d=1,2, \ldots, N-1
\end{array}\right.
\end{aligned}
$$

where the definitions of (14b) and (15b) are used in the expression.

Following the similar process shown in the previous derivation, we can deduce (A-12) and (A-13) from (A-10) and (A-11), respectively

$$
\begin{gathered}
\left\{\begin{array}{c}
X_{d}=X_{1}+X_{1}(d-1), \quad d=1,2, \cdots, N-1 \quad \text { (A-12a) } \\
S_{i}=S_{N+1}-X_{1}(i-N-1), N+1 \leq i \leq 2 N \quad \text { (A-12b) }
\end{array}\right. \\
\left\{\begin{array}{ccc}
X_{d} & =X_{1}+X_{1}(d-1), \quad d=1,2, \cdots, N-1 \quad \text { (A-13a) } \\
S_{i} & =S_{1}-X_{1}(i-1), & i=1,2, \cdots, N-1 \quad(\text { A-13b) }
\end{array}\right.
\end{gathered}
$$

where the sequence $X_{d}$ has an identical form as shown in (A-8), but the common difference of the sequence $S_{i}$ becomes $-X_{1}$.

The indexes for expressing formulas of the sequences $S_{i}$ defined by (A-9), (A-12b) and (A-13b) can be expressed by $d_{1} \in[1,2 N-1], d_{2} \in[N+1,2 N]$ and $d_{3} \in[1, N-1]$, respectively, where $d_{1}, d_{2}$ and $d_{3}$ are sets of positive integers. When $N \geq 3$, it yields

$$
\begin{aligned}
& d_{1} \cap d_{2}=[N+1,2 N-1] \\
& d_{1} \cap d_{3}=[1, N-1]
\end{aligned}
$$

where each intersection of sets has at least two sequential elements.

Note that the common difference of the sequence $S_{i}$ is $X_{1}$ in (A-9), and $-X_{1}$ in (A-12b) and (A-13b). As (A-14) shows that at least two identical sequential elements of $S_{i}$ exist in the sequences defined by (A-9) and (A-12b), (A-9) and (A-13b); it can be deduced that their common differences are identical, which yields

$$
\begin{aligned}
X_{1} & =-X_{1}+2 \pi \\
& =0 \text { or } \pi
\end{aligned}
$$

where the $+2 \pi$ term is added with the consideration of natural period of phase. 
According to (A-15), it can be concluded that both sequences $S_{i}$ and $X_{d}$ inferred by the system of equations (A-1) and (A-2) are arithmetic sequences with a common difference of 0 or $\pi$. It follows immediately that the estimation sets $\hat{\phi}_{i}$ and $\hat{\psi}_{d}^{\text {id }}$, which satisfy the system of equations (A-1) and (A-2), are different from their true values by 0 or $\pi$ plus a constant term. Although the constant term in separable phase factors is not solvable, it is canceled when the phase difference is calculated between every two receiving channels.

It should be pointed out that the system of phase calibration equations with an odd number of total elements also has the property of the fixed $\pi$-ambiguity. The proof can be carried out following a similar process.

\section{ACKNOWLEDGMENT}

The authors would like to thank the anonymous editor and reviewers for their constructive comments and suggestions. This collaboration was also supported by the Joint Ph.D. Training Program from the University of Chinese Academy of Sciences.

\section{REFERENCES}

[1] F. T. Ulaby, R. K. Moore, and A. K. Fung, Microwave Remote Sensing Active and Passive-Volume I: Microwave Remote Sensing Fundamentals and Radiometry. Artech House, 1981.

[2] Y. H. Kerr, P. Waldteufel, J. Wigneron, J. Martinuzzi, J. Font, and M. Berger, "Soil moisture retrieval from space: the Soil moisture and Ocean salinity (SMOS) mission," IEEE Transactions on Geoscience and Remote Sensing, vol. 39, no. 8, pp. 1729-1735, Aug. 2001.

[3] J. Font, G. S. E. Lagerloef, D. M. L. Vine, A. Camps, and O. Zanife, "The determination of surface salinity with the european SMOS space mission," IEEE Transactions on Geoscience and Remote Sensing, vol. 42, no. 10, pp. 2196-2205, Oct. 2004.

[4] C. D. Rodgers, "Retrieval of atmospheric temperature and composition from remote measurements of thermal radiation," Reviews of Geophysics, vol. 14, no. 4, pp. 609-624, Nov. 1976.

[5] T. T. Wilheit, "An algorithm for retrieving water vapor profiles in clear and cloudy atmospheres from $183 \mathrm{GHz}$ radiometric measurements: Simulation studies," Journal of Applied Meteorology, vol. 29, no. 6, pp. 508-515, June 1990.

[6] D. M. Levine and J. Good, "Aperture synthesis for microwave radiometers in space," NASA Tech Memo, Aug. 1983.

[7] C. S. Ruf, C. T. Swift, A. B. Tanner, and D. M. L. Vine, "Interferometric synthetic aperture microwave radiometry for the remote sensing of the earth," IEEE Transactions on Geoscience and Remote Sensing, vol. 26, no. 5, pp. 597-611, Sep. 1988.

[8] X. Bosch-Lluis, I. Ramos-Pérez, A. Camps, N. Rodriguez-Alvarez, E. Valencia, and H. Park, "Common mathematical framework for real and synthetic aperture by interferometry radiometers," IEEE Transactions on Geoscience and Remote Sensing, vol. 52, no. 1, pp. 38-50, Jan. 2014.

[9] J. Font, A. Camps, A. Borges, M. Martín-Neira, J. Boutin, N. Reul, Y. H. Kerr, A. Hahne, and S. Mecklenburg, "SMOS: The challenging sea surface salinity measurement from space," Proceedings of the IEEE, vol. 98, no. 5, pp. 649-665, May 2010.

[10] A. B. Tanner, W. J. Wilson, B. H. Lambrigsten, S. J. Dinardo, S. T. Brown, P. P. Kangaslahti, T. C. Gaier, C. S. Ruf, S. M. Gross, B. H. Lim, S. B. Musko, S. A. Rogacki, and J. R. Piepmeier, "Initial results of the geostationary synthetic thinned array radiometer (GeoSTAR) demonstrator instrument," IEEE Transactions on Geoscience and Remote Sensing, vol. 45, no. 7, pp. 1947-1957, July 2007.

[11] T. Gaier, P. Kangaslahti, B. Lambrigtsen, I. Ramos-Perez, A. Tanner, D. McKague, C. Ruf, M. Flynn, Z. Zhang, R. Backhus, and D. Austerberry, "A $180 \mathrm{GHz}$ prototype for a geostationary microwave imager/sounder-GeoSTAR-III," in 2016 IEEE International Geoscience and Remote Sensing Symposium (IGARSS), Beijing, China, July 2016, pp. 2021-2023.
[12] J. Christensen, A. Carlstrom, H. Ekstrom, A. Emrich, J. Embretsen, P. d. Maagt, and A. Colliander, "GAS: the geostationary atmospheric sounder," in 2007 IEEE International Geoscience and Remote Sensing Symposium, Barcelona, Spain, July 2007, pp. 223-226.

[13] C. Zhang, H. Liu, J. Wu, S. Zhang, J. Yan, L. Niu, W. Sun, and H. Li, "Imaging analysis and first results of the geostationary interferometric microwave sounder demonstrator," IEEE Transactions on Geoscience and Remote Sensing, vol. 53, no. 1, pp. 207-218, Jan. 2015.

[14] X. Guo, H. Liu, L. Niu, C. Zhang, H. Lu, C. Huo, T. Wang, and J. Wu, "Data processing and experimental performance of GIMS-II (Geostationary Interferometric Microwave Sounder-Second Generation) demonstrator," in 2018 IEEE 15th Specialist Meeting on Microwave Radiometry and Remote Sensing of the Environment (MicroRad), Cambridge, MA, USA, Mar. 2018, pp. 1-4.

[15] B. Lambrigtsen, "Observing clouds, convection and precipitation with a geostationary microwave sounder," in IGARSS 2019 - 2019 IEEE International Geoscience and Remote Sensing Symposium, Yokohama, Japan, July 2019, pp. 7548-7551.

[16] Z.-Q. Liu, H. Qiu, X. Li, and S.-L. Yang, "Review of large spacecraft deployable membrane antenna structures," Chinese Journal of Mechanical Engineering, vol. 30, no. 6, pp. 1447-1459, Nov. 2017. [Online]. Available: https://doi.org/10.1007/s10033-017-0198-x

[17] I. Yanovsky, B. H. Lambrigtsen, A. B. Tanner, and L. A. Vese, "Efficient deconvolution and super-resolution methods in microwave imagery," IEEE Journal of Selected Topics in Applied Earth Observations and Remote Sensing, vol. 8, no. 9, pp. 4273-4283, Sept. 2015.

[18] I. Yanovsky and B. Lambrigtsen, "Temporal super-resolution of microwave remote sensing images," in 2018 IEEE 15th Specialist Meeting on Microwave Radiometry and Remote Sensing of the Environment (MicroRad), Cambridge, MA, USA, Mar. 2018, pp. 1-6.

[19] I. Corbella, F. Torres, A. Camps, A. Colliander, M. Martin-Neira, S. Ribo, K. Rautiainen, N. Duffo, and M. Vall-llossera, "MIRAS end-toend calibration: application to SMOS L1 processor," IEEE Transactions on Geoscience and Remote Sensing, vol. 43, no. 5, pp. 1126-1134, Apr. 2005.

[20] M. A. Brown, F. Torres, I. Corbella, and A. Colliander, "SMOS calibration," IEEE Transactions on Geoscience and Remote Sensing, vol. 46, no. 3, pp. 646-658, Mar. 2008.

[21] F. Torres, A. Camps, J. Bara, I. Corbella, and R. Ferrero, "On-board phase and modulus calibration of large aperture synthesis radiometers: study applied to MIRAS," IEEE Transactions on Geoscience and Remote Sensing, vol. 34, no. 4, pp. 1000-1009, July 1996.

[22] M. H. Wieringa, "An investigation of the telescope based calibration methods 'redundancy' and 'self-cal'," Experimental Astronomy, vol. 2, no. 4, pp. 203-225, July 1992.

[23] F. R. Schwab, "Adaptive Calibration Of Radio Interferometer Data," in 1980 Intl Optical Computing Conf I, W. T. Rhodes, Ed., vol. 0231, International Society for Optics and Photonics. Washington, D.C., United States: SPIE, Aug. 1980, pp. $18-25$.

[24] Synthesis Imaging in Radio Astronomy II, ser. Astronomical Society of the Pacific Conference Series, vol. 180, Jan. 1999.

[25] J. E. Noordam and A. G. de Bruyn, "High dynamic range mapping of strong radio sources, with application to 3C84," Nature, vol. 299, no. 5884, pp. 597-600, Oct. 1982.

[26] J. S. Dillon and A. R. Parsons, "Redundant array configurations for 21 cm Cosmology," The Astrophysical Journal, vol. 826, no. 2, p. 181, Aug. 2016.

[27] H. Zheng et al., "MITEoR: a scalable interferometer for precision $21 \mathrm{~cm}$ cosmology," Monthly Notices of the Royal Astronomical Society, vol. 445, no. 2, pp. 1084-1103, Oct. 2014.

[28] A. R. Parsons et al., "The precision array for probing the epoch of reionization: Eight station results," The Astronomical Journal, vol. 139, p. 1468, Mar. 2010.

[29] D. R. DeBoer et al., "Hydrogen epoch of reionization array (HERA)," Publications of the Astronomical Society of the Pacific, vol. 129, p. 045001, Mar. 2017.

[30] P. W. Gorham, A. M. Ghez, S. R. Kulkarni, T. Nakajima, G. Neugebauer, J. B. Oke, and T. A. Prince, "Diffraction-limited imaging. III. 30 MAS closure phase imaging of six binary stars with the hale $5 \mathrm{~m}$ telescope," The Astronomical Journal, vol. 98, p. 1783, Nov. 1989.

[31] P. Negrete-Regagnon, "Practical aspects of image recovery by means of the bispectrum," Journal of the Optical Society of America A, vol. 13, no. 7, pp. 1557-1576, July 1996.

[32] J. C. Marron, P. P. Sanchez, and R. C. Sullivan, "Unwrapping algorithm for least-squares phase recovery from the modulo $2 \pi$ bispectrum phase," Journal of the Optical Society of America A, vol. 7, no. 1, pp. 14-20, Jan. 1990. 
[33] C. A. Haniff, "Least-squares fourier phase estimation from the modulo $2 \pi$ bispectrum phase," Journal of the Optical Society of America A, vol. 8, no. 1, pp. 134-140, Jan. 1991.

[34] P. Negrete-Regagnon, "Phase recovery from the bispectrum aided by the error-reduction algorithm," Optics Letters, vol. 21, no. 4, pp. 275-277, 1996.

[35] A. Liu, M. Tegmark, S. Morrison, A. Lutomirski, and M. Zaldarriaga, "Precision calibration of radio interferometers using redundant baselines," Monthly Notices of the Royal Astronomical Society, vol. 408, no. 2, pp. 1029-1050, July 2010.

[36] A. Camps, "Application of interferometric radiometry to earth observation," Ph.D. dissertation, Universitat Politècnica de Catalunya, Barcelona, Spain, 1996. [Online]. Available: "https://www.tdx.cat/handle/10803/6885"

[37] A. Camps, F. Torres, P. Lopez-Dekker, and S. J. Frasier, "Redundant space calibration of hexagonal and Y-shaped beamforming radars and interferometric radiometers," International Journal of Remote Sensing, vol. 24 , no. 24 , pp. 5183-5196, 2003.

[38] R. Dávila, F. Torres, N. Duffo, I. Corbella, M. Pablos, and M. MartínNeira, "Phase error assessment of MIRAS/SMOS by means of the redundant space calibration," in 2011 IEEE International Geoscience and Remote Sensing Symposium, Vancouver, BC, July 2011, pp. 37563759.

[39] H. Lu, Q. Li, R. Jin, K. Chen, Y. Li, L. Feng, H. Li, and Y. Li, "A hybrid calibration method for aperture synthesis radiometers," IEEE Geoscience and Remote Sensing Letters, vol. 13, no. 5, pp. 651-655, May 2016.

[40] F. Torres, A. B. Tanner, S. T. Brown, and B. H. Lambrigsten, "Analysis of array distortion in a microwave interferometric radiometer: Application to the GeoSTAR project," IEEE Transactions on Geoscience and Remote Sensing, vol. 45, no. 7, pp. 1958-1966, July 2007.

[41] I. Ramos-Perez, G. F. Forte, A. Camps, X. Bosch-Lluis, E. Valencia, N. Rodriguez-Alvarez, H. Park, and M. Vall-llosera, "Calibration, performance, and imaging tests of a fully digital synthetic aperture interferometer radiometer," IEEE Journal of Selected Topics in Applied Earth Observations and Remote Sensing, vol. 5, no. 3, pp. 723-734, June 2012.

[42] A. K. S. E. Maghraby, H. Park, A. Camps, A. Grubišić, C. Colombo, and A. Tatnall, "Phase and baseline calibration for microwave interferometric radiometers using beacons," IEEE Transactions on Geoscience and Remote Sensing, pp. 1-12, Aug. 2020.

[43] C. Zhang, J. Wu, and H. Liu, "Clock scanning microwave interferometric radiometer and potential application analysis," IEEE Journal of Selected Topics in Applied Earth Observations and Remote Sensing, vol. 8, no. 9, pp. 4262-4272, May 2015.

[44] R. Jin, Q. Li, K. Chen, G. Yi, H. Yang, D. Li, and J. Dong, "An onboard external calibration method for aperture synthesis radiometer by rotation," IEEE Geoscience and Remote Sensing Letters, vol. 9, no. 5, pp. 901-905, Sept. 2012

[45] D. Han, H. Liu, J. Wu, Z. Cheng, L. Niu, and Y. Zhang, "Interelement phase calibration for the geostationary interferometric microwave sounder (GIMS)," IEEE Geoscience and Remote Sensing Letters, vol. 13, no. 9, pp. 1216-1220, Sept. 2016.

[46] I. Corbella, N. Duffo, M. Vall-llossera, A. Camps, and F. Torres, "The visibility function in interferometric aperture synthesis radiometry," IEEE Transactions on Geoscience and Remote Sensing, vol. 42, no. 8, pp. 1677-1682, Aug. 2004.

[47] A. Camps, J. Bara, I. C. Sanahuja, and F. Torres, "The processing of hexagonally sampled signals with standard rectangular techniques: application to 2-D large aperture synthesis interferometric radiometers," IEEE Transactions on Geoscience and Remote Sensing, vol. 35, no. 1 pp. 183-190, Jan. 1997.

[48] C. Zhang, J. Wu, and W. Sun, "Applications of pseudo-polar FFT in synthetic aperture radiometer imaging," J PIERS online, vol. 3, no. 1, pp. 25-30, 2007.

[49] A. K. S. E. Maghraby, A. Grubišić, C. Colombo, and A. Tatnall, "A hexagonal pseudo-polar FFT for formation-flying interferometric radiometry," IEEE Geoscience and Remote Sensing Letters, vol. 16, no. 3, pp. 432-436, Mar. 2019.

[50] A. Camps, I. Corbella, F. Torres, M. Vall-llossera, and N. Duffo, "Polarimetric formulation of the visibility function equation including cross-polar antenna patterns," IEEE Geoscience and Remote Sensing Letters, vol. 2, no. 3, pp. 292-295, July 2005.

[51] A. Camps, I. Corbella, F. Torres, N. Duffo, M. Vall-llosera, and M. Martin-Neira, "The impact of antenna pattern frequency dependence in aperture synthesis microwave radiometers," IEEE Transactions on Geoscience and Remote Sensing, vol. 43, no. 10, pp. 2218-2224, Oct. 2005.
[52] J. Wu, C. Zhang, H. Liu, and J. Yan, "Performance analysis of circular antenna array for microwave interferometric radiometers," IEEE Transactions on Geoscience and Remote Sensing, vol. 55, no. 6, pp. 3261-3271, June 2017.

[53] A. Camps, I. Corbella, J. Bara, and F. Torres, "Radiometric sensitivity computation in aperture synthesis interferometric radiometry," IEEE Transactions on Geoscience and Remote Sensing, vol. 36, no. 2, pp. 680-685, Mar. 1998.

[54] X. Guo, A. Camps, H. Park, H. Liu, C. Zhang, and J. Wu, "Phase and amplitude calibration of equispaced circular array for geostationary passive microwave observations-Simulation results and discussion," IEEE Transactions on Geoscience and Remote Sensing, manuscript submitted for publication.

[55] G. R. Ayers, M. J. Northcott, and J. C. Dainty, "Knox-Thompson and triple-correlation imaging through atmospheric turbulence," Journal of the Optical Society of America A, vol. 5, no. 7, pp. 963-985, July 1988.

[56] E. Moore, "On the reciprocal of the general algebraic matrix," Bulletin of American Mathematical Society, vol. 26, no. 9, pp. 394-395, 1920.

[57] F. Caliò, A. Lazzari, and S. Esculapio, Elements of Mathematics with Numerical Applications. Esculapio, 2017.

[58] T. F. Coleman and Y. Li, "On the convergence of interior-reflective newton methods for nonlinear minimization subject to bounds," Mathematical Programming, vol. 67, no. 1, pp. 189-224, Oct. 1994.

[59] — , "An interior trust region approach for nonlinear minimization subject to bounds," SIAM Journal on Optimization, vol. 6, no. 2, pp. 418-445, 1996

[60] S. Kirkpatrick, C. D. Gelatt, and M. P. Vecchi, "Optimization by simulated annealing," Science, vol. 220, no. 4598, p. 671, May 1983.

[61] D. E. Goldberg, Genetic Algorithms in Search, Optimization and Machine Learning. Addison-Wesley Longman Publishing Co., Inc., 1989. 


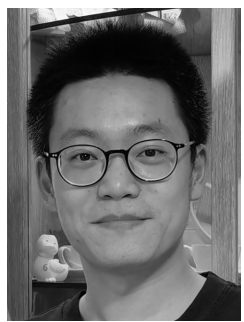

Xi Guo (Student Member, IEEE) was born in Taiyuan, China, in 1993. He received the B.S. degree in electronic engineering from Beijing Institute of Technology, Beijing, China, in 2015. He is currently pursuing the Ph.D. degree with the National Space Science Center, Chinese Academy of Sciences.

From 2019 to 2020, he was with the Remote Sensing Group, Polytechnic University of Catalonia, Barcelona, Spain, as a visiting Ph.D student. His research interests include passive remote sensing, with special emphasis on the calibration and imaging method of geostationary interferometric radiometers.

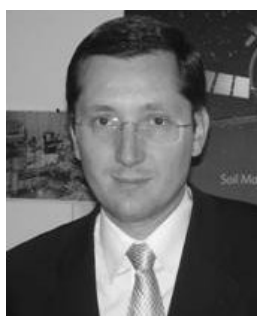

Adriano Camps (Fellow, IEEE) was born in Barcelona, Spain, in 1969. He received the degree in telecommunications engineering and the Ph.D. degree in telecommunications engineering from the Universitat Politècnica de Catalunya (UPC), Barcelona, Spain, in 1992 and 1996, respectively.

In 1993, he joined the Electromagnetics and Photonics Engineering Group, Department of Signal Theory and Communications, UPC, as an Assistant Professor, Associate Professor in 1997, and Full Professor since 2007. In 1999, he was on sabbatical leave at the Microwave Remote Sensing Laboratory, of the University of Massachusetts, Amherst. Since 1993, he has been deeply involved in the European Space Agency Soil Moisture and Ocean Salinity (SMOS) Earth Explorer Mission, from the instrument and algorithmic points of view, performing field experiments, and since 2001 studying the use of Global Navigation Satellite Systems-Reflectometry (GNSS-R) techniques to perform the sea state correction needed to retrieve salinity from L-band radiometric observations.

His research interests are focused in microwave remote sensing, with special emphasis in microwave radiometry by aperture synthesis techniques (MIRAS instrument onboard ESA's SMOS mission), remote sensing using signals of opportunity (GNSS-R), and nanosatellites as a tool to test innovative remote sensors. He has published over 227 papers in peer-reviewed journals, 8 book chapters and the book Emery and Camps, "Introduction to Satellite Remote Sensing. Atmosphere, Ocean, Land and Cryosphere Applications," Elsevier, 2017, 860 pages), and more than 472 conference presentations, holds 10 patents, and has advised $27 \mathrm{Ph}$. D. Thesis students (+ 8 on-going), and more than 140 final projects and M.Eng. Theses. According to Google Scholar/Scopus, his h-index is 52/40. He has received several awards, among which the European Young Investigator Award (2004), the ICREA Academia research award $(2009,2015)$, and the Duran Farell award for Technology Transfer $(2000,2010)$.

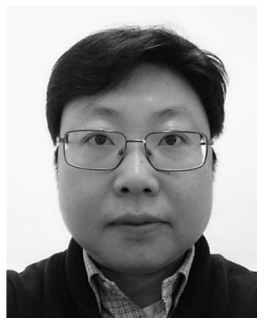

Hyuk Park (S'05-AM'09-M'12-SM'15) was born in South Korea. He received the B.S. degree in mechanical engineering from the Korea Advanced Institute of Science and Technology (KAIST), Daejeon, Korea, in 2001 and the M.S. and Ph.D. degrees in information and mechatronics from Gwangju Institute of Science and Technology (GIST), Gwangju, Korea, in 2003 and 2009, respectively. In 2009, he joined the Remote Sensing Group, Polytechnic University of Catalonia (UPC), Barcelona, Spain, as a Postdoctoral Researcher. In 2011, he was a grant holder of National Research Foundation funded by the Korean Government Since 2012, he has been working as a Research Associate with a Juan de la Cierva grant funded by the Spanish Ministry of Economy and Competitiveness. He is the winner of Chong-Hoon Cho Academic Award 2019 in Korea. $\mathrm{He}$ is currently working with School of Telecommunications and Aerospace Engineering at Castelldefels (EETAC-UPC) as a Ramon y Cajal Fellow / Tenure-track Assistant Professor. He is also working with the Passive Remote Sensing Group, UPC, for satellite remote sensing for microwave radiometry and Global Navigation Satellite Systems reflectometry. His main research interest is in the area of remote sensing, particularly passive microwave remote sensing, including system design, modeling and simulation, image I data processing, and small satellite applications.

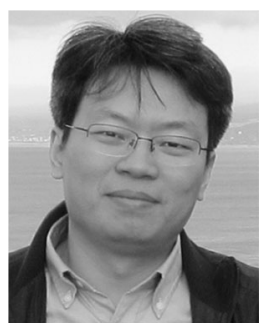

Hao Liu (A'07-M'09) was born in Jiujiang, China. $\mathrm{He}$ received the B.S. degree in information science and electronic engineering from Zhejiang University, Hangzhou, China, in 1999 and the M.S. degree in aircraft design and the Ph.D. degree in applied computer technology both from the Graduate University of Chinese Academy of Sciences, Beijing, China, in 2002 and 2011, respectively.

In 2005, he was an International Trainee with the European Space Agency. He is currently a Research Professor with the CAS Key Laboratory of Microwave Remote Sensing, National Space Science Center, Chinese Academy of Sciences, Beijing. His main research interests include microwave radiometry, particularly microwave interferometric radiometers (MIRs). His current research interests include the prestudy and demonstration of MIR systems for future geostationary millimeter-wave atmospheric sounding mission and L-band ocean salinity mission.

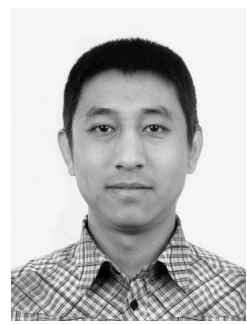

Cheng Zhang (M'10) received the B.E. degree in electronic engineering and the M.S. degree in electronic and information engineering from Hebei University of Technology, Tianjin, China, in 2001 and 2004, respectively, and the Ph.D. degree in applied computer technology from the Graduate University of Chinese Academy of Sciences (CAS), Beijing, China, in 2007.

From 2007, he has been with the CAS Key Laboratory of Microwave Remote Sensing, National Space Science Center, CAS, where he was first an Assistant Professor and has been a full Professor since 2018. His research interests include antenna array design, numerical analysis and image processing for microwave synthetic aperture interferometric radiometer.

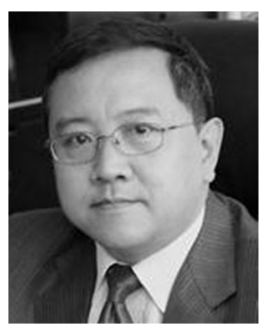

Ji Wu (S'89-M'93-SM'98-F'15) was born in Beijing, China. He received the B.S. and M.S. degrees from the Beijing University of Posts and Telecommunications, Beijing, China, and the Ph.D. degree from the Technical University of Denmark, Copenhagen, Denmark, in 1993.

From 1985 to 1986 , he was with the European Space Research and Technology Centre, Noordwijk, The Netherlands, with a United Nations Research Fellowship, where he worked on contoured beam satellite antenna design. From 1986 to 1989 , he was with the Beijing University of Posts and Telecommunications, where he worked on antenna array optimization theory and design. From 19891994, he was with Technical University of Denmark, Lyngby, Denmark, as Ph.D. student and Postdoctoral Researcher. Since 1995, he has been with the CAS Key Laboratory of Microwave Remote Sensing, National Space Science Center, Chinese Academy of Sciences, Beijing. His current research interests include interferometric imaging technology for radiometer, laser space telecommunication and space science. 